\title{
Transapical transcatheter mitral valve implantation in patients with degenerated mitral bioprostheses or failed ring annuloplasty
}

\author{
Alina Zubarevich ${ }^{1}$, Marcin Szczechowicz ${ }^{1}$, Konstantin Zhigalov $^{1}$, Philipp Marx ${ }^{1}$, Alexander Lind ${ }^{2}$, \\ Rolf Alexander Jánosi ${ }^{2}$, Tienush Rassaf ${ }^{2}$, Sharaf-Eldin Shehada ${ }^{1}$, Rizwan Malik ${ }^{1}$, Markus Kamler $^{1}$, \\ Matthias Thielmann ${ }^{1}$, Bastian Schmack ${ }^{1}$, Arjang Ruhparwar ${ }^{1}$, Alexander Weymann ${ }^{1}$, Daniel Wendt ${ }^{1}$ \\ ${ }^{1}$ Department of Thoracic and Cardiovascular Surgery, West German Heart and Vascular Center, University of Duisburg-Essen, Essen, Germany; \\ ${ }^{2}$ Department of Cardiology \& Vascular Medicine, West German Heart and Vascular Center, University of Duisburg-Essen, Essen, Germany \\ Correspondence to: Alina Zubarevich, MD. Department of Thoracic and Cardiovascular Surgery, West-German Heart Center Essen, University \\ Hospital Essen Hufelandstraße 55, 45122 Essen, Germany. Email: alina.zubarevich@gmail.com.
}

\begin{abstract}
Background: We sought to evaluate the outcomes of transapical transcatheter mitral valve replacement in patients with degenerated mitral bioprostheses or failed mitral ring annuloplasty and high surgical risk for redo mitral valve procedure.

Methods: Between August 2012 and December 2020, 41 consecutive high-risk patients underwent transcatheter mitral 'valve-in-valve' (TM-ViV, $\mathrm{n}=25$ ) or 'valve-in-ring' (TM-ViR, $\mathrm{n}=16$ ) implantation at our institution. All procedures were performed in a hybrid operating theater using the SAPIEN XT/3 ${ }^{\mathrm{TM}}$ or the DIRECT FLOW MEDICAL ${ }^{\mathrm{TM}}$ prostheses. Data was collected prospectively according to MVARC criteria. Results: The logistic EuroSCORE-I was $42.3 \% \pm 20.5 \%$ (mean \pm SD), the Society of Thoracic Surgeons (STS) score was $11.9 \% \pm 10.8 \%$, and the STS/ACC-score was $7.6 \% \pm 4.2 \%$. Transcatheter mitral valve implantations were successful in all patients (100\%). Early echocardiographic examinations showed no obstruction of the left ventricular outflow tract (LVOT), no paravalvular leakage, and only trace transvalvular leakage in eight patients. There was no operative mortality; thirty-day mortality was $9.8 \%$. Survival was $72 \%$ at one year and $63 \%$ at three years. At two-year follow-up, transvalvular mean pressure gradients were $4.6 \pm 1.4 \mathrm{mmHg}$.

Conclusions: Transapical transcatheter mitral valve-in-valve or valve-in-ring implantation represents a true minimally invasive alternative to surgical redo procedures, especially in high-risk patients with failed bioprosthetic mitral valves or annuloplasty and favorable anatomy.
\end{abstract}

Keywords: Transcatheter mitral valve-in-valve (TM-ViV); transcatheter mitral valve-in-ring (TM-ViR); transapical TMVR

Submitted Apr 21, 2021. Accepted for publication Jul 29, 2021.

doi: 10.21037/acs-2021-tviv-fs-67

View this article at: https://dx.doi.org/10.21037/acs-2021-tviv-fs-67

\section{Introduction}

Redo mitral valve (MV) surgery is the current standard of care in patients presenting with degenerated bioprosthetic MV or failed MV-annuloplasty. A surgical redo mitral valve might be required in up to $35 \%$ of patients (1) and the surgery is associated with significant morbidity and mortality especially in patients with multiple comorbidities
$(2,3)$. Transcatheter mitral valve-in-valve (TM-ViV) and valve-in-ring (TM-ViR) implantation has been already emerged as a feasible alternative to $M V$ redo surgery in high-risk patients presenting with severe MV disease. Although still classified as an off-label procedure, the predefined exact circular design of surgical MV bioprostheses, their stented nature, and the perfect fluoroscopic visibility allow safe and easy implantation of transcatheter heart 
valves within the mitral position (4-6). However, within this specific TM-ViV or TM-ViR indication, the transapical approach, in contrast to the transfemoral approach, allows a shorter, more direct and coaxial route to the diseased mitral valve prosthesis or annuloplasty ring. In this study we sought to evaluate the outcomes of transapical transcatheter mitral valve implantation using the SAPIEN valve (Edwards Lifesciences, Irvine, CA, USA) or the Direct Flow Medical valve (DFM, Santa Rosa, CA, USA) in high-risk and frail patients presenting with failed MV bioprostheses or MV annuloplasty rings.

\section{Methods}

\section{Study design and population}

Between August 2012 and December 2020, forty-one consecutive high-risk patients underwent transcatheter mitral valve implantation via the transapical route at our institution.

Preoperative evaluation of the patients was performed in our interdisciplinary institutional Heart Team, which consisted of a cardiac surgeon, interventional cardiologist, and cardiac anesthesiologist. In addition, the patient's opinion and preference was respected and well-considered and was an important part of the final decision-making process. Postoperative echocardiographic assessment of the implanted device function was performed via transthoracic echocardiography (TTE) at hospital discharge.

Patients' data were collected prospectively as part of our institutional database. This database included detailed information on patients' demographics and baseline characteristics as well as their laboratory, echocardiographic, and hemodynamic parameters, intraoperative variables and early postoperative outcomes. The study was conducted according to the revised Declaration of Helsinki and according to the principle of Good Clinical Practice. The study protocol and data gathering were approved by the ethical board of our institution, and the requirement for informed consent form patients for data use has been waived. All patients signed the informed consent on followup. An active follow-up was performed via telephone interview with the patients' general practitioner (GP) and/ or the patients.

\section{TM-ViV and TM-ViR techniques}

All procedures were performed under general anesthesia in the presence of our institutional Heart Team in a specially equipped hybrid operating room, offering full functionality for cardiac catheterization, anaesthesiology, and cardiac surgery, and a cardiopulmonary bypass circuit and clinical perfusion team were kept on stand-by. The transapical access was performed as previously described by our group (7) using four pledged U-stitches (Prolene 3-0, MH needle). In brief, access to the left ventricular apex was obtained by a 4-6 cm anterolateral minithoracotomy in the fourth, fifth or sixth intercostal space. After puncturing the apex, a soft guidewire was advanced under fluoroscopic guidance into the right pulmonary vein across the diseased mitral valve. Then, via a Pigtail catheter, an Extra-Stiff wire for further guidance was exchanged for the soft wire, and a transapical sheath (18-26 F sheath depending on valve type) was advanced. The reversely crimped transcatheter valve was finally deployed under ventricular overpacing (120 bpm). The landing zone was identified mainly with fluoroscopic guidance (Figures 1,2). Device function was evaluated by transesophageal echocardiography. Heparin was administered with an intended activated clotting time (ACT) time $>250 \mathrm{~s}$.

\section{ViV and ViR sizing}

The 'valve-in-valve app', developed by Bapat et al. and the company UBQO (8) was used for design and sizing information of the pre-existing specific mitral bioprosthesis or annuloplasty ring. This app provides all data that guides proper identification of all available surgical and transcatheter valves and rings and sizing recommendations.

\section{Outcomes and definitions}

The primary endpoints were mortality at thirty days and at one year, defined as death of any cause. Secondary endpoints were postoperative outcomes according to Mitral Valve Academic Research Consortium (MVARC) (9), and other procedure and valve-related complications.

\section{Risk calculation and definition of risk groups}

The EuroSCORE calculator available online (http://www. euroscore.org) was used for both EuroSCORE-I and EuroSCORE-II calculations. The Society of Thoracic Surgeons-Predicted Risk of Mortality (STS-PROM) calculation for mitral valve procedures was performed using the STS-Score calculator (http://riskcalc.sts.org/ 

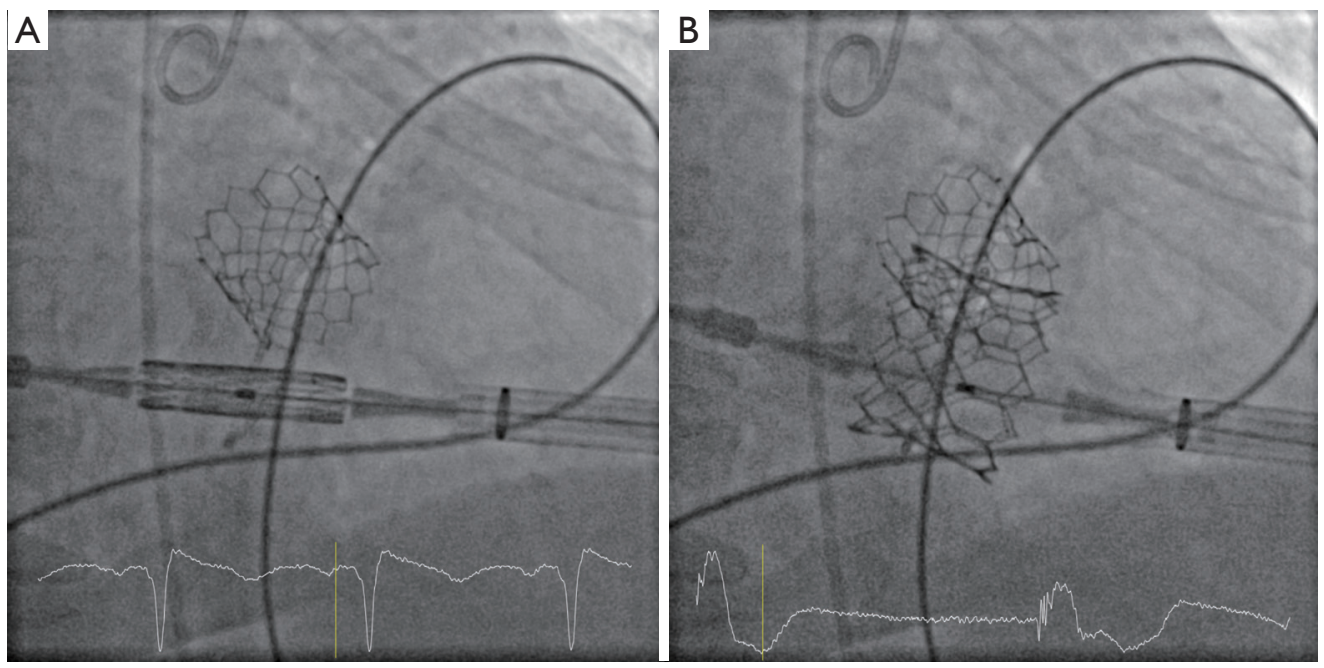

Figure 1 Valve-in-ring mitral valve implantation. (A) Positioning of the crimped transcatheter valve in the annuloplasty ring; (B) deployment of the transcatheter valve in the annuloplasty ring.

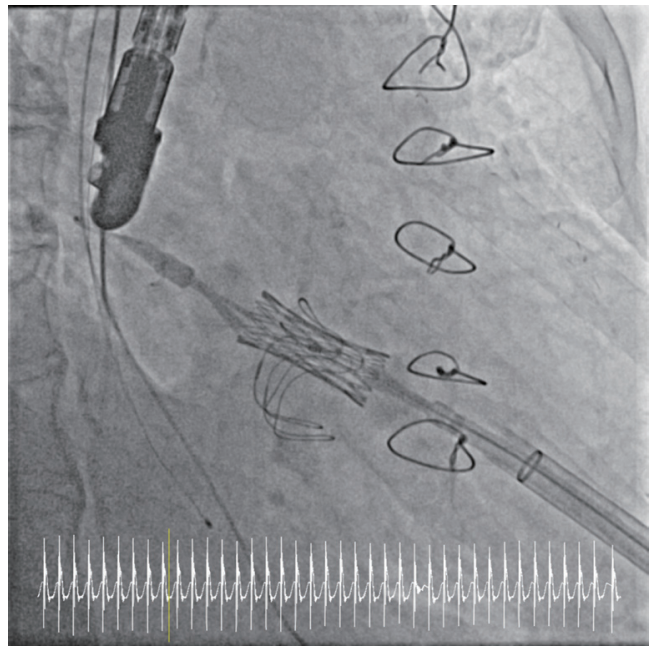

Figure 2 Valve-in valve mitral valve implantation.

stswebriskcalc/calculate) available online. The STS/ American College of Cardiology (ACC)-score transcatheter aortic valve replacement (TAVR) risk calculator app was used to calculate the STS/ACC-score (https://www.sts.org/ resources/apps). All scores were calculated for each of the forty-one patients.

\section{Statistical analysis}

The data was analyzed using IBM SPSS version 26 (IBM Corp., Chicago, IL, USA) and R software v.3.4.3 (R
Foundation for Statistical Computing, Vienna, Austria). We used the Shapiro-Wilk test to assess normality of the data. Quantitative data is presented as the mean and standard deviation (SD) or median and interquartile range (IQR), according to distribution. Categorical data is expressed as frequency and percentage. Survival curves were generated with the Kaplan-Meier method.

\section{Results}

\section{Baseline characteristics}

The mean age of the patients was $73.6 \pm 9.7$ years old (Table 1). All patients presented with symptomatic moderate to severe mitral regurgitation or moderate to severe mitral stenosis [73.2\% $(\mathrm{n}=30)$ and $48.8 \%(\mathrm{n}=20)$ respectively] and were suffering from severe dyspnea [NYHA Class III and IV 70.7\% (n=29) and 29.3\% (n=12) respectively]. All patients had previously undergone mitral valve surgery via median sternotomy and a further eleven patients (26.8\%) had received concomitant aortic valve replacement. Preoperative mean pressure gradient across the mitral valve or degenerated mitral prosthesis was $8.8 \pm 5.4 \mathrm{mmHg}$. Patients presented with multiple comorbidities as reflected by the mean logistic EuroSCORE of $42.3 \% \pm 20.5 \%$, a mean EuroSCORE-II of $21.2 \% \pm 14.8 \%$, a mean STS-Score of $7.9 \%$, and a mean ACC Score of $6.5 \%$. Severe pulmonary hypertension was present in all patients with a mean systolic pulmonary artery pressure of $63.4 \pm 16.5 \mathrm{mmHg}$. 


\begin{tabular}{|c|c|}
\hline Variable & $\mathrm{n}(\%)$ \\
\hline Female gender & $19(46.3)$ \\
\hline Age, years & $73.6 \pm 9.7$ \\
\hline Body mass index, $\mathrm{kg} / \mathrm{m}^{2}$ & $26.4 \pm 4.8$ \\
\hline NYHA III & $29(70.7)$ \\
\hline NYHA IV & $12(29.3)$ \\
\hline Systemic hypertension & $41(100.0)$ \\
\hline Pulmonary hypertension & $41(100.0)$ \\
\hline Diabetes & $14(34.1)$ \\
\hline Chronic obstructive pulmonary disease & $17(41.5)$ \\
\hline Coronary artery disease & $29(70.7)$ \\
\hline Prior percutaneous coronary intervention & $13(31.7)$ \\
\hline Peripheral vascular disease & $15(36.6)$ \\
\hline Cerebrovascular disease & $9(22.0)$ \\
\hline Prior stroke & $4(9.8)$ \\
\hline Sinus rhythm & $13(31.7)$ \\
\hline Atrial fibrillation & $28(68.3)$ \\
\hline Aortic regurgitation $\geq \|^{\circ}$ & $11(26.8)$ \\
\hline Aortic stenosis $\geq \|^{\circ}$ & $5(12.1)$ \\
\hline Mitral regurgitation (MR) & $39(95.1)$ \\
\hline$M R I^{\circ}$ & $9(22.0)$ \\
\hline MR II-III & $30(73.2)$ \\
\hline Mitral stenosis $\geq \|^{\circ}$ & $20(48.8)$ \\
\hline \multicolumn{2}{|l|}{ Tricuspid regurgitation (TR) } \\
\hline $\mathrm{TR} \leq I^{\circ}$ & $16(39.0)$ \\
\hline $\mathrm{TR} \geq \mathrm{II}^{\circ}$ & $25(61.0)$ \\
\hline Mitral valve mean gradient, $\mathrm{mmHg}$ & $8.8 \pm 5.4$ \\
\hline Prior sternotomy & $100(n=41)$ \\
\hline Prior mitral valve replacement & $25(61.0)$ \\
\hline Perimount & $25(61.0)$ \\
\hline $23 \mathrm{~mm}$ & $3(1.2)$ \\
\hline $25 \mathrm{~mm}$ & $2(4.9)$ \\
\hline $27 \mathrm{~mm}$ & $5(12.2)$ \\
\hline $29 \mathrm{~mm}$ & $4(17.1)$ \\
\hline $31 \mathrm{~mm}$ & $11(26.8)$ \\
\hline
\end{tabular}

Table 1 (continued)

\begin{tabular}{|c|c|}
\hline Variable & $\mathrm{n}(\%)$ \\
\hline Prior mitral valve repair & $16(39.0)$ \\
\hline Annuloplasty ring & $12(29.3)$ \\
\hline Annuloplasty band & $4(9.7)$ \\
\hline Prior coronary artery bypass grafting & $25(61.0)$ \\
\hline Prior surgical aortic valve replacement & $11(26.8)$ \\
\hline Prior pacemaker implantation & $11(26.8)$ \\
\hline Systolic pulmonary arterial pressure, $\mathrm{mmHg}$ & $63.4 \pm 16.5$ \\
\hline LV-EF, \% & $45.5 \pm 13.1$ \\
\hline Chronic kidney injury & $28(68.3)$ \\
\hline Dialysis & $4(9.8)$ \\
\hline Creatinine, $\mathrm{mg} / \mathrm{dL}$ & $1.7 \pm 1.1$ \\
\hline GFR $\left(\mathrm{mL} / \mathrm{min} / 1.73 \mathrm{~m}^{2}\right)$ & $44.6 \pm 19.3$ \\
\hline \multicolumn{2}{|l|}{ Preoperative anticoagulation } \\
\hline Aspirin & $26(63.4)$ \\
\hline Clopidogrel & $6(14.6)$ \\
\hline Ticagrelor & $1(2.4)$ \\
\hline Apixaban & $5(12.2)$ \\
\hline Warfarin & $11(26.8)$ \\
\hline Logistic EuroSCORE-I, \% & $42.3 \pm 20.5$ \\
\hline EuroSCORE II, \% & $21.2 \pm 14.8$ \\
\hline STS-Score, \% & $11.9 \pm 10.8$ \\
\hline ACC-Score, \% & $7.6 \pm 4.2$ \\
\hline
\end{tabular}

Data are presented as number (\%). ACC, American College of Cardiology; GFR, glomerular filtration rate; LV-EF, left ventricular ejection fraction; New York Heart Failure; STS, Society of Thoracic Surgeons.

\section{Intraoperative characteristics and postoperative outcomes}

All procedures were performed via the transapical route. Intraoperative variables are listed in Table 2. In four cases we used the Direct Flow Medical ${ }^{\mathrm{TM}}$ (DFM, Santa Rosa, CA, USA) valve prosthesis and in the remaining cases the Sapien $\mathrm{XT}^{\mathrm{TM}}$ or Sapien $3^{\mathrm{TM}}$ (Edwards Lifesciences, Irvine, CA, USA) transcatheter heart valve. A TM-ViV was performed in twenty-five and a TM-ViR procedure in sixteen patients. The mean operating time was $82.8 \pm 26.1 \mathrm{~min}$, and the mean dose of contrast was $40.0 \mathrm{~mL}$ (IQR 0-75.0 mL). 


\begin{tabular}{|ll}
\hline Table 2 Intraoperative characteristics & \\
\hline Characteristics & $\mathrm{n}(\%)$ \\
\hline Transapical access & $41(100.0)$ \\
\hline Valve-in-valve & $25(61.0)$ \\
\hline Valve-in-ring & $16(39.0)$ \\
\hline Concomitant transapical transcatheter & $8(19.5)$ \\
aortic valve implantation & \\
\hline Operating time, min & $82.8 \pm 26.1$ \\
\hline Contrast dye, mL & $40.0(\mathrm{IQR} 0-75.0)$ \\
\hline Fluoroscopy time, min & $14.1 \pm 11.8$ \\
\hline Valve prostheses & \\
\hline SapienXT/3 & $37(90.2)$ \\
\hline 29 mm & $28(68.2)$ \\
26 mm & $8(19.5)$ \\
23 mm & $1(2.4)$ \\
\hline Direct Flow Medical & $4(9.8)$ \\
29 mm & $1(2.4)$ \\
27 mm & $2(4.8)$ \\
\hline Postdilatation & $1(2.4)$ \\
\hline & $2(4.8)$ \\
\hline
\end{tabular}

Data are presented as number (\%). IQR, interquartile range.

Postoperative outcomes are listed in Table 3. There was no valve embolization, no second valve was necessary, and all transcatheter valve prostheses were successfully deployed as planned, resulting in a procedural success rate of $100 \%$. The postoperative mean gradient after transapical MV implantation at hospital discharge was $3.9 \pm 1.0 \mathrm{mmHg}$ and no paravalvular leakage or relevant (more than trace) MV regurgitation could be identified. We observed no postoperative $\mathrm{AV}$-Block $\mathrm{III}^{\circ}$ or stroke in our cohort. In addition, no relevant left ventricular outflow tract (LVOT) obstruction was detected by echocardiographic evaluation. Acute kidney injury requiring temporary dialysis occurred in $9.8 \%(n=4)$ of the patients. We found no significant difference in the major adverse events after TM-ViV vs. TM-ViR procedures.

\section{Survival}

Operative, in-hospital and thirty-day mortality, were $0 \%$,

\begin{tabular}{|ll|}
\hline Table 3 Postoperative characteristics & \\
\hline Characteristics & $\mathrm{n}(\%)$ \\
\hline Paravalvular leakage & 0 \\
\hline Rest trace MI & $8(19.5)$ \\
\hline Postoperative mean gradient, mmHg & $3.9 \pm 1.0$ \\
\hline Postoperative mean gradient at & $4.6 \pm 1.4$ \\
follow-up, mmHg & $41(100.0)$ \\
\hline Procedural success & 0 \\
\hline Dislocation & 0 \\
\hline Conversion to sternotomy & $4(9.8)$ \\
\hline Acute kidney failure with dialysis & $1(2.4)$ \\
\hline Exploration or bleeding & 0 \\
\hline Stroke & 0 \\
\hline Vascular complications & 0 \\
\hline Pacemaker implantation & 0 \\
\hline Myocardial infarction & $1(2.4)$ \\
\hline Re-intubation & $1.0($ IQR 0.75-1.0) \\
\hline Time on respirator, days & 2.0 (IQR 1.0-2.0) \\
\hline Time on intensive care unit, days & $9.7 \pm 5.4$ \\
\hline In-hospital stay, days & $3(7.3)$ \\
\hline In-hospital mortality & $4(9.8)$ \\
\hline 30-day mortality & 771 (IQR 206-1,212.0) \\
\hline Follow-up time, days & $28 \%$ \\
\hline 1-year mortality & $37 \%$ \\
\hline 3-years mortality & \\
\hline
\end{tabular}

$7.3 \%$ and $9.8 \%$, respectively. The mean follow-up time was 771 days (IQR 206-1,212 days). The survival rate for all patients was $72 \%$ at one year and $63 \%$ at three years. The Kaplan-Meier overall survival curve is given in Figure 3.

\section{Discussion}

In the present study, a total of forty-one high-risk patients presenting with failed bioprosthetic mitral valve or mitral valve annuloplasty were treated by transapical transcatheter mitral valve-in-valve or valve-in-ring implantation. This study provides a number of interesting findings:

(I) Transapical TM-ViV/TM-ViR is a viable, safe and uncomplicated treatment option that, despite 


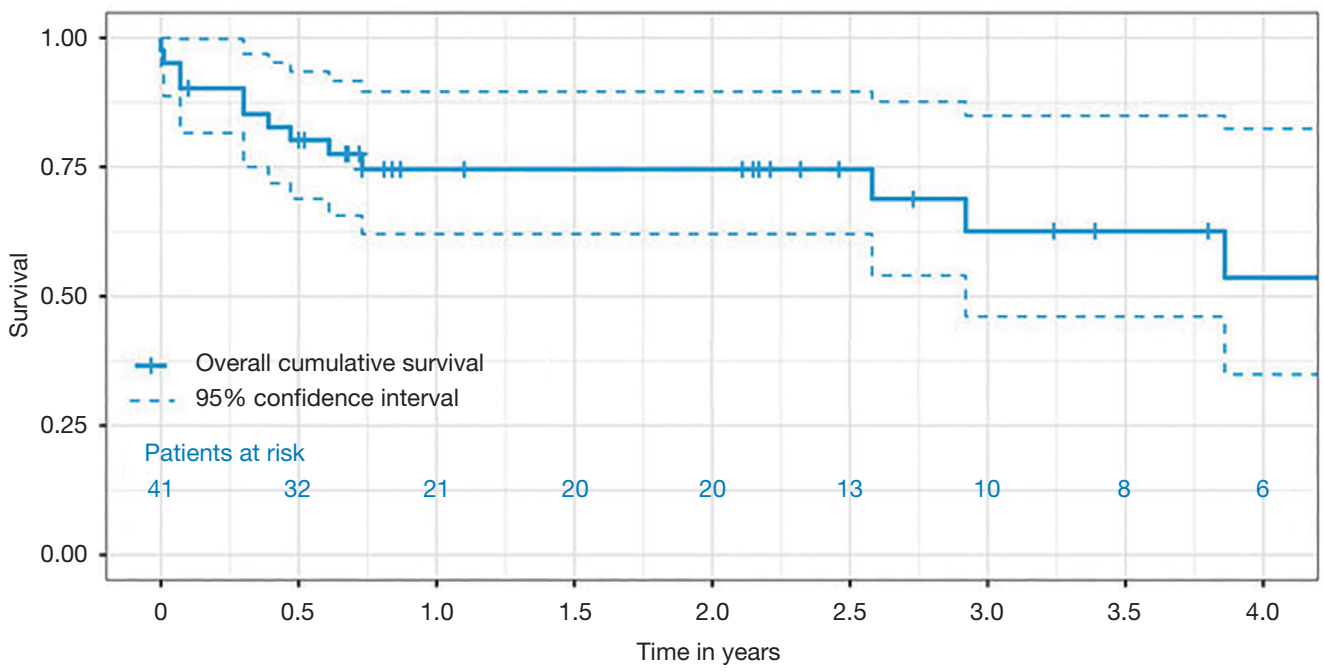

Figure 3 Overall survival.

its off-label indication, is at least a non-inferior alternative to conventional surgical redo procedures in selected high-risk patients. Transapical TMViV/TM-ViR offers high procedural success with short operating times and hospital-stay.

(II) In this presented cohort and according to our current experience, no postoperative pacemaker implantation was needed.

(III) The TM-ViV/TM-ViR procedure can be performed with a small amount of contrast medium or even without it in some cases, thus reducing the periprocedural rate of contrast-induced kidney disease. The 'mid-term' survival after transapical $\mathrm{TM}-\mathrm{ViV} / \mathrm{TM}-\mathrm{ViR}$ in high-risk patients is $63 \%$ after three years follow-up.

(IV) Transapical TM-ViV/TM-ViR provides excellent hemodynamic performance with low transvalvular gradients at 'mid-term' and a low risk of LVOT obstruction.

(V) We observed no differences in regard to postoperative major adverse events comparing TM-ViV to TM-ViR.

Following the great success of transcatheter aortic valve replacement, TM-ViV or TM-ViR implantation has also recently emerged as an alternative to conventional MV redo procedures. Therefore, we sought to evaluate our results with transapical transcatheter mitral valve replacement (TM-VR) in high-risk patients with prohibitive surgical risk. The observation period in the present study was 8 years.

According to the most recent data from The STS
National Database, the mean age of patients undergoing surgical MV redo procedures is sixty-four years (3). In the present study, however, mean age was higher (73.6 \pm 9.7 years), and all patients were at highest risk (mean logistic EuroSCORE-I $42.3 \% \pm 20.5 \%$ and STS-Score $11.9 \% \pm 10.8 \%)$. In regard to mortality, we report an inhospital mortality of $7.3 \%$ and a thirty-day mortality of $9.8 \%$. The thirty-day mortality observed in the present study is slightly lower as compared with the current literature reporting a thirty-day mortality of $13.6 \%$ (10). Compared to the percutaneous trans-septal approach, thirty-day mortality is inherently higher, as transapical patients usually come with more comorbidities such as peripheral artery disease $(11,12)$. Moreover, in a study by Eleid et al., patients undergoing the valve-in-ring procedures were excluded from their analysis, as those patients seem to carry a higher periprocedural risk, namely paravalvular leakage and LVOT obstruction (12). Despite the fact that in our cohort $39 \%$ of the patients had a history of MV annuloplasty, we observed no relevant postoperative LVOT obstruction as measured by transthoracic echocardiography. In regard to 'mid-term'-mortality, we report a one-year mortality rate of $28 \%$, which correlates with the results of Ludwig et al. in patients undergoing transapical transcatheter mitral valve replacement (5), with a survival of $63 \%$ after three years of follow-up.

Compared to surgical redo procedures, the overall procedural time of transcatheter mitral procedures is significantly lower with a mean procedural time of $82.8 \pm 26.1$ minutes in the present analysis, as also previously 
described by Simonetto et al. and Kamioka et al. $(11,13)$.

Moreover, in regard to postoperative factors, mechanical ventilation time is also an important and independent predictor for mortality in patients undergoing cardiac procedures (14) especially in the elderly. In our cohort the mean ventilation time was one day (IQR $0.75-1.0$ ) and the mean intensive care unit (ICU) stay was two days (IQR 1.0-2.0), which correlates with the current literature $(10,11)$. In our cohort, more than $40 \%$ of our patients suffered from severe chronic obstructive pulmonary disease (COPD). Therefore, most recently we changed our anesthetic strategy and we switched from endotracheal intubation to the use of a laryngeal mask, and only short-acting opioids (remifentanil) were used.

Others have shown excellent hemodynamic results for transseptal mitral valve-in-valve procedures (15). In our cohort we observed a mean transvalvular gradient of $3.9 \pm$ $1.0 \mathrm{mmHg}$ at discharge, and $4.6 \pm 1.4 \mathrm{mmHg}$ during followup, with no obstruction of the LVOT. During follow-up, we observed no prosthesis dislocation or other periprocedural complications regardless of the implanted transcatheter heart valve. Yoon et al. reported a 3\% risk for procedural complications in patients undergoing transcatheter mitral valve implantation for degenerated bioprostheses, failed annuloplasty rings, and mitral annular calcification. Patients with failed annuloplasty carry higher risk compared to patients undergoing a valve-in-valve procedure due to technical challenges (15). None of the patients in our cohort suffered a postoperative stroke or needed postoperative pacemaker implantation (16). We also report no specific valve related complications, despite the high proportion of patients undergoing a valve-in-ring procedure in our cohort. In our present analysis, with $39 \%$ of patients undergoing a TM-ViR procedure, we did not observe any differences in outcomes between TM-ViR and TM-ViV. Patients undoing a TM-ViV procedure present with a more "beneficial" landing zone compared to annuloplasty rings, and are therefore less likely to embolize. In the present analysis we implanted all valves under ventricular overpacing $(120 \mathrm{bpm})$ in a slow and stepwise fashion with the possibility of repositioning before final deployment.

Kidney function (pre- and postoperative) has already been described as an independent predictor of mortality in patients undergoing TAVR $(17,18)$. In the present study, almost $68 \%$ of our patients suffered from pre-operative kidney injury with four patients being on chronic dialysis. A correlation between the amount of contrast agent used and post-procedural acute kidney injury was reported by
Yamamoto et al. in patients undergoing TAVR (19). In our cohort, the median amount of contrast dye used was $40.0 \mathrm{~mL}$ (IQR 0-75.0), which is quite low and even less compared to the contrast dye amount reported by Kamioka et al. $(13,20)$. We believe that due to the fluoroscopic qualities of prior implanted surgical mitral valve prostheses and mitral rings acting as a 'docking station', the amount of contrast could be further reduced or even completely waived.

Despite these benefits of TM-ViV and TM-ViR procedures, there are still some patients in whom this approach might not be possible, namely patients presenting with endocarditis of the valve or the annuloplasty ring, or patients presenting with "open" annuloplasty-bands like the Cosgrove-band. Even patients with "closed" annuloplasty rings might not be possible to treat, as a $34 \mathrm{~mm}$ annuloplasty ring represents a borderline size for TM-ViR procedures. Moreover, the LVOT should be evaluated precisely in the pre-operative cardiac CT-scan to rule out patients at risk for LVOT obstruction.

\section{Conclusions}

In the growing era of transcatheter mitral valve implantation, transapical transcatheter mitral valve replacement also represents a true minimally invasive alternative to surgical redo procedures, especially in high-risk patients with failed bioprosthetic mitral valves or annuloplasty and favorable anatomy. However, the percutaneous approach should be kept in mind as a real alternative to redo surgery. Moreover, even in such high-risk patients, with some modifications, low postoperative complication rates and less (or even no) contrast use can be achieved. Our cohort reflects the current 'real-world' situation for high-risk patients with prohibitive surgical risk of a mitral valve redo procedure. With this in mind, transapical transcatheter mitral valve replacement can and should be used in high-risk patients, offering a one-year survival rate of more than $70 \%$. In our cohort, mortality was mainly driven by renal failure. According to our recent experience, especially with defined 'docking stations' like valves or annuloplasty rings, the amount of contrast can be dramatically reduced. Moreover, we did not observe any differences in outcomes comparing TM-ViR and TM-ViV.

\section{Study limitations}

This study is limited by its retrospective, non-randomized singe-center nature and a short follow-up time. Moreover, 
we summarize a rather small number of high-risk patients and encompass the experience of a single center; therefore, the generalizability of our findings may not extend to all of the clinical centers performing transcatheter mitral valve procedures. So far, only a few studies with smaller singlecenter cohorts on this topic have been published. Further prospective studies on larger cohorts should be conducted to validate the safety and efficiency of this method.

\section{Acknowledgments}

Funding: None.

\section{Footnote}

Conflicts of Interest: Daniel Wendt is working as a proctor for Edwards Lifesciences. The other authors have no conflicts of interest.

Open Access Statement: This is an Open Access article distributed in accordance with the Creative Commons Attribution-NonCommercial-NoDerivs 4.0 International License (CC BY-NC-ND 4.0), which permits the noncommercial replication and distribution of the article with the strict proviso that no changes or edits are made and the original work is properly cited (including links to both the formal publication through the relevant DOI and the license). See: https://creativecommons.org/licenses/by-nc-nd/4.0/.

\section{References}

1. Vohra HA, Whistance RN, Roubelakis A, et al. Outcome after redo-mitral valve replacement in adult patients: a 10year single-centre experience. Interact Cardiovasc Thorac Surg 2012;14:575-9.

2. Mehaffey HJ, Hawkins RB, Schubert S, et al. Contemporary outcomes in reoperative mitral valve surgery. Heart 2018;104:652-6.

3. Kilic A, Acker MA, Gleason TG, et al. Clinical Outcomes of Mitral Valve Reoperations in the United States: An Analysis of The Society of Thoracic Surgeons National Database. Ann Thorac Surg 2019;107:754-9.

4. Whisenant B, Kapadia SR, Eleid MF, et al. One-Year Outcomes of Mitral Valve-in-Valve Using the SAPIEN 3 Transcatheter Heart Valve. JAMA Cardiol 2020;5:1245-52.

5. Ludwig S, Kalbacher D, Schofer N, et al. Early results of a real-world series with two transapical transcatheter mitral valve replacement devices. Clin Res Cardiol
2021;110:411-20.

6. Wilbring M, Alexiou K, Tugtekin SM, et al. Transapical transcatheter valve-in-valve implantation for deteriorated mitral valve bioprostheses. Ann Thorac Surg 2013;95:111-7.

7. Wendt D, Al-Rashid F, Kahlert P, et al. Conventional aortic valve replacement or transcatheter aortic valve implantation in patients with previous cardiac surgery. J Cardiol 2015;66:292-7.

8. Bapat V. Valve-in-valve apps: why and how they were developed and how to use them. EuroIntervention 2014;10 Suppl U:U44-51.

9. Stone GW, Vahanian AS, Adams DH, et al. Clinical Trial Design Principles and Endpoint Definitions for Transcatheter Mitral Valve Repair and Replacement: Part 1: Clinical Trial Design Principles: A Consensus Document From the Mitral Valve Academic Research Consortium. J Am Coll Cardiol 2015;66:278-307.

10. Del Val D, Ferreira-Neto AN, Wintzer-Wehekind J, et al. Early Experience With Transcatheter Mitral Valve Replacement: A Systematic Review. J Am Heart Assoc 2019;8:e13332.

11. Simonetto F, Purita PAM, Malerba M, et al. Surgical redo versus transseptal or transapical transcatheter mitral valvein-valve implantation for failed mitral valve bioprosthesis. Catheter Cardiovasc Interv 2021;97:714-22.

12. Eleid MF, Whisenant BK, Cabalka AK, et al. Early Outcomes of Percutaneous Transvenous Transseptal Transcatheter Valve Implantation in Failed Bioprosthetic Mitral Valves, Ring Annuloplasty, and Severe Mitral Annular Calcification. JACC Cardiovasc Interv 2017;10:1932-42.

13. Kamioka N, Babaliaros V, Morse MA, et al. Comparison of Clinical and Echocardiographic Outcomes After Surgical Redo Mitral Valve Replacement and Transcatheter Mitral Valve-in-Valve Therapy. JACC Cardiovasc Interv 2018;11:1131-8.

14. Fernandez-Zamora MD, Gordillo-Brenes A, BanderasBravo E, et al. Prolonged Mechanical Ventilation as a Predictor of Mortality After Cardiac Surgery. Respir Care 2018;63:550-7.

15. Yoon SH, Whisenant BK, Bleiziffer S, et al. Outcomes of transcatheter mitral valve replacement for degenerated bioprostheses, failed annuloplasty rings, and mitral annular calcification. Eur Heart J 2019;40:441-51.

16. Wendt D, Shehada SE, König L, et al. Modified implantation height of the Sapien $3^{\mathrm{TM}}$ transcatheter heart valve. Minim Invasive Ther Allied Technol 2020;29:70-7. 
17. Sinning JM, Ghanem A, Steinhäuser H, et al. Renal function as predictor of mortality in patients after percutaneous transcatheter aortic valve implantation. JACC Cardiovasc Interv 2010;3:1141-9.

18. Lanz J, Kim WK, Walther T, et al. Safety and efficacy of a self-expanding versus a balloon-expandable bioprosthesis for transcatheter aortic valve replacement in patients with symptomatic severe aortic stenosis: a randomised noninferiority trial. Lancet 2019;394:1619-28.

Cite this article as: Zubarevich A, Szczechowicz M, Zhigalov K, Marx P, Lind A, Jánosi RA, Rassaf T, Shehada SE, Malik R, Kamler M, Thielmann M, Schmack B, Ruhparwar A, Weymann A, Wendt D. Transapical transcatheter mitral valve implantation in patients with degenerated mitral bioprostheses or failed ring annuloplasty. Ann Cardiothorac Surg 2021;10(5):674-682. doi: 10.21037/acs-2021-tviv-fs-67
19. Yamamoto M, Hayashida K, Mouillet G, et al. Renal function-based contrast dosing predicts acute kidney injury following transcatheter aortic valve implantation. JACC Cardiovasc Interv 2013;6:479-86.

20. Zubarevich A, Zhigalov K, Szczechowicz M, et al. Simultaneous transaortic transcatheter aortic valve implantation and off-pump coronary artery bypass: An effective hybrid approach. J Card Surg 2021;36:1226-31. 